\title{
SET-VALUED LEBESGUE AND RIESZ TYPE THEOREMS
}

\author{
BY
}

\author{
ANCA PRECUPANU and ALINA GAVRILUT
}

\begin{abstract}
In this paper, we continue a previous study concerning different types of pseudo-convergences of sequences of measurable functions with respect to set-valued nonadditive monotonic set functions and we establish some pseudo-versions of Lebesgue and Riesz theorems in the set-valued case. We also characterize some important structural properties of fuzzy multimeasures.

Mathematics Subject Classification 2000: 28B20, 28C15.

Key words: continuous from below, continuous from above, null-additive, pseudoautocontinuous, pseudo-order continuous, pseudo-almost everywhere convergence, pseudoalmost uniform convergence, pseudo-convergence in measure, Lebesgue type theorems, Riesz type theorem.
\end{abstract}

\section{Introduction}

As it is well-known, convergence theorems for sequences of measurable functions play a very important role in classical measure theory.

Relationships among different types of convergences such as almost everywhere convergence, almost uniform convergence and convergence in measure were especially described by the fundamental results contained in the Egoroff, Lebesgue and Riesz theorems (Precupanu [19]).

In non-additive measure theory, we mention the remarkable contributions of Wang and Klir [30], PAP [18], Denneberg [1], Li and YASuda [13], Li [9, 10], Li and Li [11], Li et AL. [14], Murofushi ET AL. [17], KaWABE [6, 7] concerning Egoroff's theorem, the papers of Li [9], Song and Li [24] for Lebesgue's theorem or Sun [25] for Riesz's theorem and Jiang et AL. [5] or TAKahashi et AL. [27], Ha ET Al. [3], LiU [15], Li ET AL. [12], Li [9], Li ET AL. [14], Li ET AL. [8], concerning different 
convergence theorems of sequences of measurable functions. We also remark the papers of Murofushi [16], Ren et al. [23], Sun [26], Zhang [28], WANG [29] and many others.

Recently, motivated by the applied problems coming from mathematic economics, artificial intelligence, biomathematics and other important fields, some of the above mentioned results were generalized in the set-valued case. In this sense, we remark the paper of LIU [15], in which are given setvalued versions of Egoroff theorem and of Lebesgue theorem for sequences of set-valued measurable functions, our papers [20-22] concerning Egoroff and Lusin theorems for set-valued fuzzy multimeasures, or the paper of $\mathrm{Wu}$ and LIU [31], which contains a set-valued version of Riesz theorem.

The aim of this paper is to continue for set-valued non-additive monotonic set functions, the investigation concerning different types of convergences and pseudo-convergences of sequences of measurable functions. Thus, we give several set-valued versions of Lebesgue theorem and a pseudoversion of Riesz's theorem, in which intercomes the property (PS) introduced by us in the set-valued case.

\section{Terminology and notations}

Let $T$ be an abstract space, $\mathcal{A}$ a $\sigma$-algebra of subsets of $T, X$ a real normed space with the origin $0, \mathcal{P}_{0}(X)$ the family of all nonvoid subsets of $X, \mathcal{P}_{f}(X)$ the family of closed, nonvoid sets of $X, \mathcal{P}_{b f}(X)$ the family of all bounded, closed, nonvoid sets of $X, \mathcal{P}_{b f c}(X)$ the family of all bounded, closed, convex nonvoid sets of $X$ and $h$ the Hausdorff pseudometric on $\mathcal{P}_{f}(X)$ given by:

$$
h(M, N)=\max \{e(M, N), e(N, M)\}, \text { for every } M, N \in \mathcal{P}_{f}(X),
$$

where $e(M, N)=\sup _{x \in M} d(x, N)$ is the excess of $M$ over $N$.

It is known that $e(M, N)=0$ if and only if $M \subset N$. Therefore, $e(M, N)=$ $h(M, N)$, for every $M, N \in \mathcal{P}_{f}(X)$, with $N \subset M$. Also, $e(M, N) \leq e(M, P)+$ $e(P, N)$, for every $M, N, P \in \mathcal{P}_{f}(X)$. On $\mathcal{P}_{b f}(X), h$ becomes a metric [4].

We denote $|M|=h(M,\{0\})$, for every $M \in \mathcal{P}_{f}(X)$. We also denote $A \cap$ $\mathcal{A}=\{E \subset A, E \in \mathcal{A}\}$, where $A$ is a fixed set in $\mathcal{A}$.

Throughout the paper we shall use the following notions in the set valued case:

Definition 2.1 ([2], [20]-[22]). A set multifunction $\mu: \mathcal{A} \rightarrow \mathcal{P}_{f}(X)$ is said to be: 
i) a fuzzy multimeasure if $\mu$ is monotone with respect to the inclusion of sets (i.e., $\mu(A) \subseteq \mu(B)$, for every $A, B \in \mathcal{A}$, with $A \subseteq B$ ) and $\mu(\emptyset)=\{0\}$.

ii) continuous from below if $\lim _{n \rightarrow \infty} h\left(\mu\left(A_{n}\right), \mu(A)\right)=0$, for every increasing sequence of sets $\left(A_{n}\right)_{n} \subset \mathcal{A}$, with $A_{n} \nearrow A$.

iii) continuous from above if $\lim _{n \rightarrow \infty} h\left(\mu\left(A_{n}\right), \mu(A)\right)=0$, for every decreasing sequence of sets $\left(A_{n}\right)_{n} \subset \mathcal{A}$, with $A_{n} \searrow A$.

iv) a fuzzy multimeasure in the sense of Sugeno, for short (S)-fuzzy multimeasure, if $\mu$ is a fuzzy multimeasure which is continuous from below and continuous from above.

v) order continuous if $\lim _{n \rightarrow \infty}\left|\mu\left(A_{n}\right)\right|=0$, for every sequence of sets $\left(A_{n}\right)_{n} \subset \mathcal{A}$, with $A_{n} \searrow \emptyset$.

vi) strongly order continuous if $\lim _{n \rightarrow \infty}\left|\mu\left(A_{n}\right)\right|=0$, for every sequence of sets $\left(A_{n}\right)_{n} \subset \mathcal{A}$, with $A_{n} \searrow A$ and $\mu(A)=\{0\}$.

vii) pseudo-order continuous if for every $B \in \mathcal{A}$ and every sequence of sets $\left(A_{n}\right)_{n} \subset \mathcal{A}$ with $A_{n} \subset B, n \in \mathbb{N}$ and $A_{n} \searrow A$, we have $\lim _{n \rightarrow \infty}\left|\mu\left(A_{n}\right)\right|=0$ and $\mu(B \backslash A)=\mu(B)$.

viii) null-additive if $\mu(A \cup B)=\mu(B)$, for every disjoint $A, B \in \mathcal{A}$, with $\mu(A)=\{0\}$.

ix) pseudo-null-additive if $\mu(B \cup C)=\mu(C)$, whenever $A \in \mathcal{A}, B \in A \cap$ $\mathcal{A}, C \in A \cap \mathcal{A}$ and $\mu(A \backslash B)=\mu(A)$.

$\mathrm{x})$ a) autocontinuous from below (autocontinuous from above, respectively) if for every $A \in \mathcal{A}$ and every $\left(B_{n}\right)_{n} \subset \mathcal{A}$, with $\lim _{n \rightarrow \infty}\left|\mu\left(B_{n}\right)\right|=0$, we have $\lim _{n \rightarrow \infty} h\left(\mu\left(A \backslash B_{n}\right), \mu(A)\right)=0\left(\lim _{n \rightarrow \infty} h\left(\mu\left(A \cup B_{n}\right), \mu(A)\right)=0\right.$, respectively).

b) autocontinuous if it is autocontinuous from above and autocontinuous from below.

xi) a) pseudo-autocontinuous from above (pseudo-autocontinuous from below, respectively) if for every $A \in \mathcal{A}$ and every $\left(B_{n}\right)_{n} \subset \mathcal{A}$, with $\lim _{n \rightarrow \infty} h\left(\mu\left(B_{n} \cap A\right), \mu(A)\right)=0$, we have $\lim _{n \rightarrow \infty} h\left(\left(\mu\left(A \backslash B_{n}\right) \cup C\right), \mu(C)\right)$ $=0$ (respectively, $\lim _{n \rightarrow \infty} h\left(\left(\mu\left(B_{n} \cap C\right), \mu(C)\right)=0\right)$, for every $C \in$ $A \cap \mathcal{A}$. 
b) pseudo-autocontinuous if it is pseudo-autocontinuous from above and pseudo-autocontinuous from below.

Definition 2.2. We say that a set multifunction $\mu: \mathcal{A} \rightarrow \mathcal{P}_{f}(X)$ fulfils:

i) $[20,22]$ property $(S)$ if for any sequence of sets $\left(A_{n}\right)_{n} \subset \mathcal{A}$, with $\lim _{n \rightarrow \infty}\left|\mu\left(A_{n}\right)\right|=0$, there exists a subsequence $\left(A_{n_{k}}\right)_{k}$ of $\left(A_{n}\right)_{n}$ such that $\mu\left(\varlimsup_{\lim _{k}} A_{n_{k}}\right)=\{0\}$, where $\varlimsup_{n} E_{n}=\lim \sup _{n} E_{n}=\bigcap_{n=1}^{\infty} \bigcup_{k=n}^{\infty} E_{k}$.

ii) [22] property $(P S)$ if for any $A \in \mathcal{A}$ and any sequence of sets $\left(A_{n}\right)_{n} \subset$ $A \cap \mathcal{A}$, with $\lim _{n \rightarrow \infty} h\left(\mu\left(A_{n}\right), \mu(A)\right)=0$, there exists a subsequence $\left(A_{n_{k}}\right)_{k}$ of $\left(A_{n}\right)_{n}$ such that $h\left(\mu\left(\underline{\lim }_{k} A_{n_{k}}\right), \mu(A)\right)=0$, where $\underline{\lim }_{n} E_{n}=$ $\liminf _{n} E_{n}=\bigcup_{n=1}^{\infty} \bigcap_{k=n}^{\infty} E_{k}$.

Unless stated otherwise, all over the paper we assume that $\mu: \mathcal{A} \rightarrow \mathcal{P}_{f}(X)$ is a fuzzy (i.e., monotone) multimeasure. By $\mathcal{M}$ we denote the class of all $\mathcal{A}$-measurable real-valued functions on $(T, \mathcal{A}, \mu)$, the space with the fuzzy multimeasure $\mu$.

Definition 2.3. We consider arbitrary $\left\{f_{n}\right\} \subset \mathcal{M}$ and $f \in \mathcal{M}$. We say that:

i) [22] $\left\{f_{n}\right\}$ converges $\mu$-almost everywhere (respectively, pseudo- $\mu$-almost everywhere) to $f$ on $A$, and denote it by $f_{n} \underset{A}{\stackrel{a . e}{\rightarrow}} f$ (respectively, $\left.f_{n} \underset{A}{\stackrel{p . a . e .}{\longrightarrow}} f\right)$ if there exists a subset $B \in A \cap \mathcal{A}$ such that $\mu(B)=\{0\}$ (respectively, $\mu(A \backslash B)=\mu(A))$ and $\left\{f_{n}\right\}$ is pointwise convergent to $f$ on $A \backslash B$.

ii) [22] $\left\{f_{n}\right\}$ converges in $\mu$-measure (respectively, pseudo in $\mu$-measure) to $f$ on $A$, and denote it by $f_{n} \underset{A}{\stackrel{\mu}{\longrightarrow}} f$ (respectively, $f_{n} \underset{A}{\stackrel{p \cdot \mu}{\longrightarrow}} f$ ) if for every $\varepsilon>0, \lim _{n \rightarrow \infty} \mid \mu\left(A_{n}(\varepsilon) \mid=0\right.$, where $A_{n}(\varepsilon)=\left\{t \in A ; \mid f_{n}(t)-\right.$ $f(t) \mid \geq \varepsilon\}$ (respectively, $\left.\lim _{n \rightarrow \infty} h\left(\mu\left(A \backslash A_{n}(\varepsilon)\right), \mu(A)\right)=0\right)$.

iii) $[20,22]\left\{f_{n}\right\}$ converges $\mu$-almost uniformly (respectively, $\mu$-pseudoalmost uniformly) to $f$ on $A$ and denote it by $f_{n} \underset{A}{\stackrel{a . u_{u}}{\longrightarrow}} f$ (respectively, $\left.f_{n} \underset{A}{\stackrel{p . a . u .}{\longrightarrow}} f\right)$ if there exists a decreasing sequence $\left\{A_{k}\right\}_{k \in \mathbb{N}} \subset A \cap \mathcal{A}$ such that $\lim _{k \rightarrow \infty}\left|\mu\left(A_{k}\right)\right|=0$ (respectively, $\lim _{k \rightarrow \infty} h\left(\mu\left(A \backslash A_{k}\right), \mu(A)\right)=$ $0)$ and for every fixed $k \in \mathbb{N},\left\{f_{n}\right\}$ uniformly converges to $f$ on $A \backslash A_{k}$ $\left(f_{n} \underset{A \backslash A_{k}}{\stackrel{u}{\longrightarrow}} f\right)$. 
Remark 2.4 ([22]). The following statements are equivalent:

a) $\mu$ is pseudo-null-additive;

b) $\mu(B \cap C)=\mu(C)$, whenever $A \in \mathcal{A}, B \in A \cap \mathcal{A}, C \in A \cap \mathcal{A}$ and $\mu(B)=\mu(A)$

c) $\mu((A \backslash B) \cup C)=\mu(C)$, whenever $A \in \mathcal{A}, B \in A \cap \mathcal{A}, C \in A \cap \mathcal{A}$ and $\mu(B)=\mu(A)$.

\section{Set-valued versions of Lebesgue theorems}

In this section, we present some set-valued versions of Lebesgue theorem.

Firstly, using some ideas from [9] and [24], we establish several set-valued versions of Lebesgue theorem. In this way, we give some characterizations for several important asymptotic structural properties of monotone set multifunctions.

Theorem 3.1 (Lebesgue type). Let be $A \in \mathcal{A}, f \in \mathcal{M}$ and $\left\{f_{n}\right\} \subset \mathcal{M}$. Then:

i) $f_{n} \underset{A}{\stackrel{a . e .}{\rightarrow}} f \Rightarrow f_{n} \underset{A}{\stackrel{\mu}{\longrightarrow}} f$ if and only if $\mu$ is strongly order continuous;

ii) $f_{n} \underset{A}{\stackrel{p . a . e .}{\rightarrow}} f \Rightarrow f_{n} \underset{A}{\stackrel{p . \mu}{\rightarrow}} f$ if and only if $\mu$ is continuous from below;

iii) $f_{n} \underset{A}{\stackrel{\text { p.a.e. }}{\rightarrow}} f \Rightarrow f_{n} \underset{A}{\stackrel{\mu}{\longrightarrow}} f$ if and only if $\mu$ is pseudo-order continuous;

iv) If $\mu: \mathcal{A} \rightarrow \mathcal{P}_{b f}(X)$, then $f_{n} \underset{A}{\stackrel{a . e .}{\rightarrow}} f \Rightarrow f_{n} \underset{A}{\stackrel{p . \mu}{\rightarrow}} f$ if and only if $\mu$ is null-additive and continuous from below.

Proof. Take arbitrary $A \in \mathcal{A}$ and $f \in \mathcal{M},\left\{f_{n}\right\} \subset \mathcal{M}$. We observe that the set $C$ of points $t \in A$ at which $\left\{f_{n}\right\}$ is pointwise convergent to $f$ can be written as $C=\bigcap_{m=1}^{\infty} \bigcup_{n=1}^{\infty} \bigcap_{i=n}^{\infty}\left(A \backslash B_{i}\left(\frac{1}{m}\right)\right)$, where $B_{i}\left(\frac{1}{m}\right)=$ $\left\{t \in A ;\left|f_{i}(t)-f(t)\right| \geq \frac{1}{m}\right\}$, for every $m, i \in \mathbb{N}^{*}$. For every $m, n \in \mathbb{N}^{*}$, we denote $A_{n}^{(m)}=\bigcup_{i=n}^{\infty} B_{i}\left(\frac{1}{m}\right)$ and $A^{(m)}=\bigcap_{n=1}^{\infty} A_{n}^{(m)}$. Then $A \backslash A^{(m)}=$ $\bigcup_{n=1}^{\infty}\left(A \backslash A_{n}^{(m)}\right)=\bigcup_{n=1}^{\infty} \bigcap_{i=n}^{\infty}\left(A \backslash B_{i}\left(\frac{1}{m}\right)\right)$ and $C=\bigcap_{m=1}^{\infty} \bigcup_{n=1}^{\infty}\left(A \backslash A_{n}^{(m)}\right)=$ $\bigcap_{m=1}^{\infty}\left(A \backslash A^{(m)}\right)$. We observe that for every fixed $m \in \mathbb{N}^{*}, A_{n}^{(m)} \underset{n \rightarrow \infty}{\searrow} A^{(m)}$ 
and so, $A \backslash A_{n}^{(m)} \underset{n \rightarrow \infty}{\nearrow} A \backslash A^{(m)}$. If there exists a set $B \in A \cap \mathcal{A}$ and $\left\{f_{n}\right\}$ is pointwise convergent to $f$ on $A \backslash B$, then for every $m \in \mathbb{N}^{*}$,

$$
A \backslash B \subset C \subset \bigcup_{n=1}^{\infty} \bigcap_{i=n}^{\infty}\left(A \backslash B_{i}\left(\frac{1}{m}\right)\right)=A \backslash A^{(m)} \subset A .
$$

We also observe that

$$
B_{n}\left(\frac{1}{m}\right) \subset A_{n}^{(m)}, \text { for every } m, n \in \mathbb{N}^{*} .
$$

i) Necessity. To prove that $\mu$ is strongly order continuous, let us consider $\left(A_{n}\right)_{n \in \mathbb{N}} \subset A \cap \mathcal{A}$, with $A_{n} \searrow \widetilde{A}$ and $\mu(\widetilde{A})=\{0\}$. Then $\widetilde{A} \subset A_{n} \subset A$, for every $n \in \mathbb{N}$. We shall prove that $\lim _{n \rightarrow \infty}\left|\mu\left(A_{n}\right)\right|=0$. For every $n \in \mathbb{N}$, we define the following functions:

$$
f_{n}(t)= \begin{cases}0, & \text { if } t \in A_{n} \\ 1, & \text { if } t \in A \backslash A_{n}\end{cases}
$$

and

$$
f(t)= \begin{cases}0, & \text { if } t \in \widetilde{A} \\ 1, & \text { if } t \in A \backslash \widetilde{A} .\end{cases}
$$

We observe that $f \in \mathcal{M},\left\{f_{n}\right\} \subset \mathcal{M}$ and $\left\{f_{n}\right\}$ is pointwise convergent to 1 on $A \backslash \widetilde{A}$, so $f_{n} \underset{A}{\stackrel{a . e_{\text {. }}}{A}} 1$. By virtue of hypothesis, $f_{n} \underset{A}{\stackrel{\mu}{\rightarrow}} f$, whence $\lim _{n \rightarrow \infty}\left|\mu\left(\left\{t \in A ;\left|f_{n}(t)-1\right| \geq \frac{1}{2}\right\}\right)\right|=0$, which implies that $\lim _{n \rightarrow \infty}\left|\mu\left(A_{n}\right)\right|$ $=0$. This means that $\mu$ is strongly order continuous.

Sufficiency. Suppose $\mu$ is strongly order continuous and $f_{n} \underset{A}{\stackrel{a . e .}{\rightarrow}} f$. Then there exists $B \in A \cap \mathcal{A}$ such $\mu(B)=\{0\}$ and $\left\{f_{n}\right\}$ is pointwise convergent to $f$ on $A \backslash B$. By (1), for every $m \in \mathbb{N}^{*}, A^{(m)} \subset B$ and so, because $\mu$ is a fuzzy multimeasure, we get that $\mu\left(A^{(m)}\right)=\{0\}$.

Since for every fixed $m \in \mathbb{N}^{*}, A_{n}^{(m)} \underset{n \rightarrow \infty}{\searrow} A^{(m)}$ and $\mu$ is strongly order continuous, then for every $m \in \mathbb{N}^{*}, \lim _{n \rightarrow \infty}\left|\mu\left(A_{n}^{(m)}\right)\right|=0$. By (2), we get that for every $m \in \mathbb{N}^{*}, \lim _{n \rightarrow \infty}\left|\mu\left(B_{n}\left(\frac{1}{m}\right)\right)\right|=0$, that is, $f_{n} \underset{A}{\stackrel{\mu}{\longrightarrow}} f$.

ii) Necessity: To prove that $\mu$ is continuous from below, let us consider $\left(A_{n}\right)_{n \in \mathbb{N}} \subset A \cap \mathcal{A}$, with $A_{n} \nearrow \widetilde{A}$. Then $A_{n} \subset \widetilde{A} \subset A$, for every $n \in \mathbb{N}$. 
We shall prove that $\lim _{n \rightarrow \infty} h\left(\mu\left(A_{n}\right), \mu(\widetilde{A})\right)=0$. For every $n \in \mathbb{N}$, we define $f,\left\{f_{n}\right\}$ as follows:

$$
f_{n}(t)= \begin{cases}0, & \text { if } t \in A \backslash A_{n} \\ 1, & \text { if } t \in A_{n}\end{cases}
$$

and

$$
f(t)= \begin{cases}0, & \text { if } t \in A \backslash \widetilde{A} \\ 1, & \text { if } t \in \widetilde{A} .\end{cases}
$$

We observe that $f \in \mathcal{M},\left\{f_{n}\right\} \subset \mathcal{M}$ and $\left\{f_{n}\right\}$ is pointwise convergent to $f$ on $\widetilde{A}$, so $f_{n} \underset{\widetilde{A}}{\stackrel{p . a . e .}{\longrightarrow}} f$.

Consequently, $f_{n} \underset{\widetilde{A}}{\stackrel{p \cdot \mu}{\longrightarrow}} f$, whence $\lim _{n \rightarrow \infty} h\left(\mu\left(\widetilde{A} \backslash\left\{t \in \widetilde{A} ;\left|f_{n}(t)-f(t)\right| \geq\right.\right.\right.$ $\left.\left.\left.\frac{1}{2}\right\}\right), \mu(\widetilde{A})\right)=0$ and so, $\lim _{n \rightarrow \infty} h\left(\mu\left(\widetilde{A} \backslash\left(A \backslash A_{n}\right)\right), \mu(\widetilde{A})\right)=0$, which implies that $\lim _{n \rightarrow \infty} h\left(\mu\left(A_{n}\right), \mu(\widetilde{A})\right)=0$, that is, $\mu$ is continuous from below.

Sufficiency: Suppose that $\mu$ is continuous from below and $f_{n} \underset{A}{\stackrel{p . a . e .}{\longrightarrow}} f$. Then there exists $B \in A \cap \mathcal{A}$ such that $\mu(A \backslash B)=\mu(A)$ and $\left\{f_{n}\right\}$ is pointwise convergent to $f$ on $A \backslash B$. Because $\mu(A \backslash B)=\mu(A)$ and $\mu$ is a fuzzy multimeasure, by (1) we have

$$
\mu(A \backslash B)=\mu(C)=\mu\left(\bigcup_{n=1}^{\infty} \bigcap_{i=n}^{\infty}\left(A \backslash B_{i}\left(\frac{1}{m}\right)\right)\right)=\mu(A)=\mu\left(A \backslash A^{(m)}\right) .
$$

By (2), we get $\mu\left(A \backslash A_{n}^{(m)}\right) \subset \mu\left(A \backslash B_{n}\left(\frac{1}{m}\right)\right) \subset \mu(A)$. Since $\mu$ is continuous from below, then for every $m \in \mathbb{N}$, $\lim _{n \rightarrow \infty} h\left(\mu\left(A \backslash A_{n}^{(m)}\right), \mu\left(A \backslash A^{(m)}\right)\right)=0$, that is, by $(3), \lim _{n \rightarrow \infty} h\left(\mu\left(A \backslash A_{n}^{(m)}\right), \mu(A)\right)=0$.

Using Lemma 2.1 from [22], we have $\lim _{n \rightarrow \infty} h\left(\mu\left(A \backslash B_{n}\left(\frac{1}{m}\right), \mu(A)\right)=0\right.$, which says that $f_{n} \underset{A}{\stackrel{p \cdot \mu}{\rightarrow}} f$.

iii) Necessity. To prove that $\mu$ is pseudo order continuous, let us consider arbitrary $\left(A_{n}\right)_{n} \subset \mathcal{A}$ and $B \in \mathcal{A}$, with $A_{n} \subset B$, for every $n, A_{n} \searrow \widetilde{A} \in \mathcal{A}$ and $\mu(B \backslash \widetilde{A})=\mu(B)$. We shall prove that $\lim _{n \rightarrow \infty}\left|\mu\left(A_{n}\right)\right|=0$. For every $n \in \mathbb{N}$, we define $f,\left\{f_{n}\right\}$ as follows:

$$
f_{n}(t)= \begin{cases}0, & \text { if } t \in A_{n} \\ 1, & \text { if } t \in B \backslash A_{n}\end{cases}
$$


and

$$
f(t)= \begin{cases}0, & \text { if } t \in \widetilde{A} \\ 1, & \text { if } t \in B \backslash \widetilde{A} .\end{cases}
$$

We observe that $f \in \mathcal{M},\left\{f_{n}\right\} \subset \mathcal{M}$ and $\left\{f_{n}\right\}$ is pointwise convergent to 1 on $B \backslash \widetilde{A}$, so $f_{n} \underset{B}{\stackrel{\text { p.a.e. }}{\rightarrow}} 1$.

Consequently, $f_{n} \underset{B}{\stackrel{\mu}{\rightarrow}} 1$, whence $\lim _{n \rightarrow \infty}\left|\mu\left(\left\{t \in B ;\left|f_{n}(t)-1\right| \geq \frac{1}{2}\right\}\right)\right|=$ 0 , which implies $\lim _{n \rightarrow \infty}\left|\mu\left(A_{n}\right)\right|=0$, that is, $\mu$ is pseudo-order continuous.

Sufficiency. Suppose $\mu$ is pseudo-order continuous and $f_{n} \underset{A}{\stackrel{p . a . e .}{\rightarrow}} f$. There exists $B \in A \cap \mathcal{A}$ such that $\mu(A \backslash B)=\mu(A)$ and $\left\{f_{n}\right\}$ is pointwise convergent to $f$ on $A \backslash B$. By (1), we have for every $m \in \mathbb{N}^{*}, \mu(A)=\mu\left(A \backslash A^{(m)}\right)$. Consequently, because for every fixed $m \in \mathbb{N}^{*}, A_{n}^{(m)} \underset{n \rightarrow \infty}{\searrow} A^{(m)}$ and $\mu$ is pseudo-order continuous, then $\lim _{n \rightarrow \infty}\left|\mu\left(A_{n}^{(m)}\right)\right|=0$. By (2), we get that for every $m \in \mathbb{N}^{*}, \lim _{n \rightarrow \infty}\left|\mu\left(B_{n}\left(\frac{1}{m}\right)\right)\right|=0$, that is, $f_{n} \underset{A}{\longrightarrow} f$.

iv) Necessity: First, we prove that $\mu$ is continuous from below.

Let us consider the sequence $\left\{f_{n}\right\}$ from ii), which is pointwise convergent to $f$ on $\widetilde{A}$, so $f_{n} \underset{\widetilde{A}}{\stackrel{a \cdot e}{\longrightarrow}} f$. By virtue of the hypothesis, $f_{n} \underset{\widetilde{A}}{\stackrel{p \cdot \mu}{\longrightarrow}} f$, whence, as in ii), $\mu$ is continuous from below.

It only remains to prove that $\mu$ is null-additive. For this, take arbitrary disjoint $B_{1}, B_{2} \in \mathcal{A}$, with $\mu\left(B_{1}\right)=\{0\}$. For every $n \in \mathbb{N}$, we define

$$
f_{n}(t)=\left\{\begin{array}{l}
0, \text { if } t \in B_{1} \\
1, \text { if } t \in B_{2}
\end{array} .\right.
$$

Then $f_{n} \underset{B_{1} \cup B_{2}}{\stackrel{a . e_{j}}{\rightarrow}} 1$, whence $f_{n} \underset{B_{1} \cup B_{2}}{\stackrel{p . \mu}{\longrightarrow}} 1$, so, $h\left(\mu\left(\left(B_{1} \cup B_{2}\right) \backslash\left\{t \in B_{1} \cup B_{2} ; \mid f_{n}(t)-\right.\right.\right.$ $\left.\left.\left.\left.1 \mid \geq \frac{1}{2}\right\}\right)\right), \mu\left(B_{1} \cup B_{2}\right)\right)=0$, which implies $h\left(\mu\left(B_{2}\right), \mu\left(B_{1} \cup B_{2}\right)\right)=0$. Because $\mu: \mathcal{A} \rightarrow \mathcal{P}_{b f}(X)$, we finally have $\mu\left(B_{1} \cup B_{2}\right)=\mu\left(B_{2}\right)$, so, $\mu$ is null-additive.

Sufficiency: Suppose $\mu$ is null-additive and continuous from below and $f_{n} \underset{A}{\stackrel{a . e_{i}}{\rightarrow}} f$. Since $\mu$ is null-additive, we have by Proposition 3.1 from [22] that $f_{n} \underset{A}{\stackrel{p . a . e .}{\rightarrow}} f$ and by ii) we obtain that $f_{n} \underset{A}{\stackrel{p . \mu}{\rightarrow}} f$.

\section{Set-valued versions of Riesz theorem}

In this section, we present some characterizations of pseudo-autocontinuity and we establish a pseudo-version of Riesz theorem. 
Firstly, we mention the following generalization of the set-valued version of Riesz's theorem [31] and some of its consequences.

Theorem 4.1 (Riesz type theorem). $\mu$ has property $(S)$ if and only if for every $A \in \mathcal{A}$ and every $f \in \mathcal{M},\left\{f_{n}\right\} \subset \mathcal{M}$, with $f_{n} \underset{A}{\rightarrow} f$, there exists a subsequence $\left\{f_{n_{k}}\right\}_{k}$ of $\left\{f_{n}\right\}$ so that $f_{n_{k}} \underset{A}{\stackrel{\text { a.e. }}{\rightarrow}} f$.

By Theorem 3.1 i) and Theorem 4.1, we get:

Corollary 4.2. Let be $\mu$ a strongly order continuous fuzzy multimeasure which fulfils property $(S)$ and $A \in \mathcal{A}, f \in \mathcal{M},\left\{f_{n}\right\} \subset \mathcal{M}$. Then:

i) $f_{n} \underset{A}{\stackrel{\text { a.e. }}{\rightarrow}} f$ implies $f_{n} \underset{A}{\stackrel{\mu}{\longrightarrow}} f$.

ii) If $f_{n} \underset{A}{\stackrel{\mu}{\longrightarrow}} f$, there is a subsequence $\left\{f_{n_{k}}\right\}_{k}$ of $\left\{f_{n}\right\}$ so that $f_{n_{k}} \underset{A}{\stackrel{a . e .}{\rightarrow}} f$.

Since any continuous from above fuzzy multimeasure is strongly order continuous, by [20] (Corollary 4.7), and by the above considerations we get:

Remark 4.3. Let be arbitrary $A \in \mathcal{A}, f \in \mathcal{M}$ and $\left\{f_{n}\right\} \subset \mathcal{M}$ and suppose $\mu$ is continuous from above, with property (S). Then:

i) $f_{n} \underset{A}{\stackrel{a . e_{.}}{\rightarrow}} f \Leftrightarrow f_{n} \underset{A}{\stackrel{a . u}{\rightarrow}} f$;

ii) If $f_{n} \underset{A}{\stackrel{a \cdot e .}{\longrightarrow}} f$, then $f_{n} \underset{A}{\stackrel{\mu}{\longrightarrow}} f$;

iii) If $f_{n} \underset{A}{\stackrel{\mu}{\longrightarrow}} f$, there exists a subsequence $\left\{f_{n_{k}}\right\}_{k}$ of $\left\{f_{n}\right\}$ such that $f_{n_{k}} \underset{A}{\stackrel{a . e \cdot}{\rightarrow}} f$.

Using some ideas from $[25,26]$, we also prove the following characterizations for pseudo-autocontinuity:

Theorem 4.4. If $\mu: \mathcal{A} \rightarrow \mathcal{P}_{b f}(X)$ is a (S)-fuzzy multimeasure, then the following statements are equivalent:

i) $\mu$ is pseudo-autocontinuous;

ii) $\mu$ is pseudo-autocontinuous from below;

iii) $\mu$ is pseudo-autocontinuous from above; 
iv) $\mu$ is pseudo-null-additive and fulfils property $(P S)$;

v) $\mu$ is pseudo-null-additive and for every $A \in \mathcal{A}$ and every $\left(A_{n}\right)_{n} \subset$ $\mathcal{A} \cap A$, with $\lim _{n \rightarrow \infty} h\left(\mu\left(A_{n}\right), \mu(A)\right)=0$, there exists a subsequence $\left(A_{n_{k}}\right)_{k}$ so that $\mu\left(A \backslash \underline{\lim }_{k} A_{n_{k}}\right)=\{0\}$.

Proof. In order to prove that i) $\Leftrightarrow$ ii) $\Leftrightarrow$ iii), it is sufficient to prove that ii) $\Leftrightarrow$ iii):

iii) $\Rightarrow$ ii): Let us assume that $\mu$ is pseudo-autocontinuous from above, but $\mu$ is not pseudo-autocontinuous from below. Then there exist $\varepsilon_{0}>0$, $A \in \mathcal{A}, C \in A \cap \mathcal{A},\left(B_{n}\right) \subset A \cap \mathcal{A}$ so that $\lim _{n \rightarrow \infty} h\left(\mu\left(B_{n}\right), \mu(A)\right)=0$, but $h\left(\mu\left(B_{n} \cap C\right), \mu(C)\right)=e\left(\mu(C), \mu\left(B_{n} \cap C\right)\right)>\varepsilon_{0}$, for every $n \in \mathbb{N}$. Let $n_{1}=1$. Since $\mu$ is pseudo-autocontinuous from above, then $\lim _{n \rightarrow \infty} h\left(\mu\left(\left(B_{n_{1}} \cap C\right) \cup\right.\right.$ $\left.\left.\left(A \backslash B_{n}\right)\right), \mu\left(B_{n_{1}} \cap C\right)\right)=0$. Since for every $n \in \mathbb{N}$,

$$
\begin{aligned}
& \varepsilon_{0}<e\left(\mu(C), \mu\left(B_{n_{1}} \cap C\right)\right) \leq e\left(\mu(C), \mu\left(\left(B_{n_{1}} \cap C\right) \cup\left(A \backslash B_{n}\right)\right)\right) \\
& \quad+e\left(\mu\left(\left(B_{n_{1}} \cap C\right) \cup\left(A \backslash B_{n}\right)\right), \mu\left(B_{n_{1}} \cap C\right)\right),
\end{aligned}
$$

then $\lim _{n \rightarrow \infty} e\left(\mu(C), \mu\left(\left(B_{n_{1}} \cap C\right) \cup\left(A \backslash B_{n}\right)\right)\right)>\varepsilon_{0}$, so, there exists $n_{2} \in$ $\mathbb{N}$ such that $n_{2}>n_{1}$ and $e\left(\mu(C), \mu\left(\left(B_{n_{1}} \cap C\right) \cup\left(A \backslash B_{n_{2}}\right)\right)>\varepsilon_{0}\right.$. Taking again into account that $\mu$ is pseudo-autocontinuous from above, then $\lim _{n \rightarrow \infty} h\left(\mu\left(\left(B_{n_{2}} \cap C\right) \cup\left(A \backslash B_{n}\right)\right), \mu\left(B_{n_{2}} \cap C\right)\right)=0$ and $\lim _{n \rightarrow \infty} h\left(\mu\left(\left(B_{n_{1}} \cap\right.\right.\right.$ $\left.\left.C) \cup\left(A \backslash B_{n_{2}}\right) \cup\left(A \backslash B_{n}\right)\right), \mu\left(\left(B_{n_{1}} \cap C\right) \cup\left(A \backslash B_{n_{2}}\right)\right)\right)=0$, so, as before, there exists $n_{3} \in \mathbb{N}$ such that $n_{3}>n_{2}, e\left(\mu(C), \mu\left(\left(B_{n_{2}} \cap C\right) \cup\left(A \backslash B_{n_{3}}\right)\right)\right)>\varepsilon_{0}$ and $e\left(\mu(C), \mu\left(\left(B_{n_{1}} \cap C\right) \cup\left(A \backslash B_{n_{2}}\right) \cup\left(A \backslash B_{n_{3}}\right)\right)\right)>\varepsilon_{0}$.

Analogously, there exists $n_{4} \in \mathbb{N}$ such that $n_{4}>n_{3}, e\left(\mu(C), \mu\left(\left(B_{n_{3}} \cap\right.\right.\right.$ $\left.\left.C) \cup\left(A \backslash B_{n_{4}}\right)\right)\right)>\varepsilon_{0}, e\left(\mu(C), \mu\left(\left(B_{n_{2}} \cap C\right) \cup\left(A \backslash B_{n_{3}}\right) \cup\left(A \backslash B_{n_{4}}\right)\right)\right)>\varepsilon_{0}$ and $e\left(\mu(C), \mu\left(\left(B_{n_{1}} \cap C\right) \cup\left(A \backslash B_{n_{2}}\right) \cup\left(A \backslash B_{n_{3}}\right) \cup\left(A \backslash B_{n_{4}}\right)\right)\right)>\varepsilon_{0}$. Recurrently, there exists $\left(B_{n_{k}}\right)_{k} \subset\left(B_{n}\right)$ so that for every $k \in \mathbb{N}$,

$$
e\left(\mu(C), \mu\left(\left(B_{n_{k}} \cap C\right) \cup\left(A \backslash \bigcap_{t=k+1}^{\infty} B_{n_{t}}\right)\right)\right)>\varepsilon_{0} .
$$

Denote $D_{k}=B_{n_{k}} \cup\left(A \backslash \bigcap_{t=k+1}^{\infty} B_{n_{t}}\right)$, for every $k \in \mathbb{N}$.

We observe that $\liminf \inf _{k} D_{k}=\operatorname{lim\operatorname {sup}_{k}} D_{k}=A$ and, for every $k \in \mathbb{N}$, we have $\mu\left(C \cap\left(\bigcap_{t=k}^{\infty} D_{t}\right)\right) \subset \mu\left(\left(B_{n_{k}} \cap C\right) \cup\left(A \backslash \bigcap_{t=k+1}^{\infty} B_{n_{t}}\right)\right)$, so,

$$
h\left(\mu\left(C \cap\left(\bigcap_{t=k}^{\infty} D_{t}\right)\right), \mu(C)\right)=e\left(\mu(C), \mu\left(C \cap\left(\bigcap_{t=k}^{\infty} D_{t}\right)\right)\right)
$$




$$
\begin{aligned}
& =e\left(\mu(C), \mu\left(C \cap\left(\bigcap_{t=k}^{\infty} D_{t}\right)\right)\right) \\
& +e\left(\mu\left(C \cap\left(\bigcap_{t=k}^{\infty} D_{t}\right)\right), \mu\left(\left(B_{n_{k}} \cap C\right) \cup\left(A \backslash \bigcap_{t=k+1}^{\infty} B_{n_{t}}\right)\right)\right) \\
& \geq e\left(\mu(C), \mu\left(\left(B_{n_{k}} \cap C\right) \cup\left(A \backslash \bigcap_{t=k+1}^{\infty} B_{n_{t}}\right)\right)\right)>\varepsilon_{0} .
\end{aligned}
$$

Since $C \cap\left(\bigcap_{t=k}^{\infty} D_{t}\right) \underset{k \rightarrow \infty}{\nearrow} C \cap A=C$ and $\mu$ is continuous from below, then $\lim _{k \rightarrow \infty} h\left(\mu\left(C \cap\left(\bigcap_{t=k}^{\infty} D_{t}\right)\right), \mu(C)\right)=0$, whence $0 \geq \varepsilon_{0}$, which is a contradiction.

ii) $\Rightarrow$ iv) a) Firstly, we prove that $\mu$ is pseudo-null-additive.

Indeed, for every $A \in \mathcal{A}, B \in A \cap \mathcal{A}, C \in A \cap \mathcal{A}$, with $\mu(A \backslash B)=\mu(A)$, applying the pseudo-autocontinuity from below of $\mu$ for $B_{n}=A \backslash B$, for every $n \in \mathbb{N}$, we get that $h(\mu((A \backslash B) \cap C), \mu(C))=0$. Because $\mu: \mathcal{A} \rightarrow$ $\mathcal{P}_{b f}(X)$, then $\mu(C)=\mu(C \backslash B)$. Replacing $C$ by $C \cup B$, we get $\mu(C \cup B)=$ $\mu((C \cup B) \backslash B)=\mu(C \backslash B)=\mu(C)$, that is, $\mu$ is pseudo-null-additive.

b) Now, we prove that $\mu$ has PS. Suppose $A \in \mathcal{A},\left(A_{n}\right) \subset A \cap \mathcal{A}$ are so that $\lim _{n \rightarrow \infty} h\left(\mu\left(A_{n}\right), \mu(A)\right)=0$ and let be arbitrary $\varepsilon>0$. Since $\mu$ is pseudo-autocontinuous from below, we get that $\lim _{n \rightarrow \infty} h\left(\mu\left(A_{n} \cap\right.\right.$ $C), \mu(C))=0$, for every $C \in A \cap \mathcal{A}$. Since $\lim _{n \rightarrow \infty} h\left(\mu\left(A_{n}\right), \mu(A)\right)=0$, there is $n_{1} \in \mathbb{N}$ so that $h\left(\mu\left(A_{n_{1}}\right), \mu(A)\right)<\frac{\varepsilon}{2}<\varepsilon$. Because $\lim _{n \rightarrow \infty} h\left(\mu\left(A_{n} \cap\right.\right.$ $\left.\left.A_{n_{1}}\right), \mu\left(A_{n_{1}}\right)\right)=0$, there is $n_{2} \in \mathbb{N}$, so that $h\left(\mu\left(A_{n_{2}} \cap A_{n_{1}}\right), \mu\left(A_{n_{1}}\right)\right)<\frac{\varepsilon}{2^{2}}$, so $h\left(\mu\left(A_{n_{2}} \cap A_{n_{1}}\right), \mu(A)\right)<\frac{\varepsilon}{2}+\frac{\varepsilon}{2^{2}}<\varepsilon$. Analogously, also taking into account that $\mu$ is continuous from above, we get that there exists $\left(A_{n_{k}}\right)_{k} \subset\left(A_{n}\right)$ so that $h\left(\mu\left(\bigcap_{k=1}^{\infty} A_{n_{k}}\right), \mu(A)\right) \leq \sum_{k=1}^{\infty} \frac{\varepsilon}{2^{k}}=\varepsilon$.

Consequently, $e\left(\mu(A), \mu\left(\bigcap_{k=1}^{\infty} A_{n_{k}}\right)\right) \leq \varepsilon$, whence, for every $s \in \mathbb{N}^{*}$, $e\left(\mu(A), \mu\left(\bigcap_{k=s}^{\infty} A_{n_{k}}\right)\right) \leq e\left(\mu(A), \mu\left(\bigcap_{k=1}^{\infty} A_{n_{k}}\right)\right) \leq \varepsilon$.

Since $\bigcap_{k=s}^{\infty} A_{n_{k}} \nearrow \bigcup_{s \rightarrow \infty}^{\infty} \bigcap_{k=s}^{\infty} A_{n_{k}}$ and $\mu$ is continuous from below, then $e\left(\mu(A), \mu\left(\bigcup_{s=1}^{\infty} \bigcap_{k=s}^{s \rightarrow \infty} A_{n_{k}}\right)\right)=0$. Consequently, because $\mu: \mathcal{A} \rightarrow$ $\mathcal{P}_{b f}(X)$, we have $\mu(A)=\mu\left(\bigcup_{s=1}^{\infty} \bigcap_{k=s}^{\infty} A_{n_{k}}\right)=\mu\left(\liminf _{k} A_{n_{k}}\right)$, that is, $\mu$ has property (PS).

iv) $\Rightarrow$ ii) Consider arbitrary $A \in \mathcal{A},\left(B_{n}\right) \subset \mathcal{A}, C \in A \cap \mathcal{A}$, so that $\lim _{n \rightarrow \infty} h\left(\mu\left(A \cap B_{n}\right), \mu(A)\right)=0$. There exists a subsequence $\left(B_{n_{k}}\right)_{k}$ of $\left(B_{n}\right)$ so that $\limsup _{n} h\left(\mu\left(C \cap B_{n}\right), \mu(C)\right)=\lim _{k \rightarrow \infty} h\left(\mu\left(C \cap B_{n_{k}}\right), \mu(C)\right)$.

Applying property (PS) for $A \cap B_{n_{k}}$ and since $\mu: \mathcal{A} \rightarrow \mathcal{P}_{b f}(X)$, there exists $\left(B_{n_{k_{s}}} \cap A\right)_{s}$ so that $\mu\left(\liminf _{s}\left(B_{n_{k_{s}}} \cap A\right)\right)=\mu(A)$. 
Consequently, by the pseudo-null-additivity of $\mu$, according to Remark 2.4, we have $\left.\mu\left(\left(\liminf _{s}\left(B_{n_{k_{s}}} \cap A\right) \cap C\right)\right)\right)=\mu(A \cap C)$, that is, equivalently, $\mu\left(\liminf _{s}\left(B_{n_{k_{s}}} \cap C\right)\right)=\mu(C)$. On the other hand, because $\bigcap_{t=s}^{\infty} C \cap$ $B_{n_{k_{t}}} \nearrow_{s \rightarrow \infty} \liminf \operatorname{in}_{s}\left(B_{n_{k_{s}}} \cap C\right)$ and $\mu$ is continuous from below, we have

$$
\begin{aligned}
0 & \leq \limsup _{n} h\left(\mu\left(C \cap B_{n}\right), \mu(C)\right)=\lim _{s \rightarrow \infty} h\left(\mu\left(C \cap B_{n_{k_{s}}}\right), \mu(C)\right) \\
& =\lim _{s \rightarrow \infty} e\left(\mu(C), \mu\left(C \cap B_{n_{k_{s}}}\right)\right) \leq \lim _{s \rightarrow \infty}\left[e\left(\mu(C), \mu\left(\bigcap_{t=s}^{\infty}\left(C \cap B_{n_{k_{t}}}\right)\right)\right)\right. \\
& \left.+e\left(\mu\left(\bigcap_{t=s}^{\infty}\left(C \cap B_{n_{k_{t}}}\right)\right), \mu\left(C \cap B_{n_{k_{s}}}\right)\right)\right]=\lim _{s \rightarrow \infty} e\left(\mu(C), \mu\left(\bigcap_{t=s}^{\infty}\left(C \cap B_{n_{k_{t}}}\right)\right)\right) \\
& \leq \lim _{s \rightarrow \infty}\left[e\left(\mu(C), \mu\left(\bigcup_{s=1}^{\infty} \bigcap_{t=s}^{\infty}\left(C \cap B_{n_{k_{t}}}\right)\right)\right)\right. \\
& \left.+e\left(\mu\left(\bigcup_{s=1}^{\infty} \bigcap_{t=s}^{\infty}\left(C \cap B_{n_{k_{t}}}\right)\right), \mu\left(\bigcap_{t=s}^{\infty} C \cap B_{n_{k_{t}}}\right)\right)\right] \\
& =e\left(\mu(C), \mu\left(\liminf _{s}\left(B_{n_{k_{s}}} \cap C\right)\right)\right)=0,
\end{aligned}
$$

so $\lim \sup _{n} h\left(\mu\left(C \cap B_{n}\right), \mu(C)\right)=0$, whence $\lim _{n \rightarrow \infty} h\left(\mu\left(C \cap B_{n}\right), \mu(C)\right)=$ 0 , that is, $\mu$ is pseudo-autocontinuous from below.

iv) $\Rightarrow$ v) Consider arbitrary $A \in \mathcal{A},\left(A_{n}\right)_{n} \subset A \cap \mathcal{A}$, with $\lim _{n \rightarrow \infty} h\left(\mu\left(A_{n}\right)\right.$, $\mu(A))=0$. Since $\mu: \mathcal{A} \rightarrow \mathcal{P}_{b f}(X)$ has (PS), there exists a subsequence $\left(A_{n_{k}}\right)_{k} \subset\left(A_{n}\right)$ so that $\mu(A)=\mu\left(\varliminf_{k} A_{n_{k}}\right)=\mu\left(A \backslash\left(A \backslash \varliminf_{k} A_{n_{k}}\right)\right)$.

Since $\mu$ is pseudo-null-additive, then $\left.\mu\left(\left(A \backslash \varliminf_{k} A_{n_{k}}\right) \cup C\right)\right)=\mu(C)$, for every $C \in A \cap \mathcal{A}$. Particularly, for $C=\emptyset$, we get $\mu\left(A \backslash \varliminf_{k} A_{n_{k}}\right)=\{0\}$.

v) $\Rightarrow$ iv) Consider arbitrary $A \in \mathcal{A},\left(A_{n}\right)_{n} \subset A \cap \mathcal{A}$, with $\lim _{n \rightarrow \infty} h\left(\mu\left(A_{n}\right)\right.$, $\mu(A))=0$.

By the hypothesis, there exists a subsequence $\left(A_{n_{k}}\right)_{k} \subset\left(A_{n}\right)$ so that $\mu\left(A \backslash \varliminf_{k} A_{n_{k}}\right)=\{0\}$. Since $\mu$ is pseudo-null-additive, then $\mu\left(\left(A \backslash \underline{\lim }_{k} A_{n_{k}}\right) \cup\right.$ $C))=\mu(C)$, for every $C \in A \cap \mathcal{A}$. Particularly, for $C=\underline{\lim }_{k} A_{n_{k}}$, we get $\mu(A)=\mu\left(\underline{\lim }_{k} A_{n_{k}}\right)$.

It only remains to prove that $\mathrm{v}) \Rightarrow$ iii). For this, one can use the same method as in the implication iv) $\Rightarrow$ ii), and Remark 2.4.

Now, we are in position to give the following pseudo-version of Riesz's theorem: 
Theorem 4.5 (a pseudo-version of Riesz's theorem). Let be $\mu$ a fuzzy multimeasure, $A \in \mathcal{A}, f \in \mathcal{M}$ and $\left\{f_{n}\right\} \subset \mathcal{M}$ so that $f_{n} \underset{A}{\stackrel{p . \mu}{\rightarrow}} f$. Then there exists a subsequence $\left\{f_{n_{k}}\right\}_{k}$ of $\left\{f_{n}\right\}$ such that $f_{n_{k}}^{\stackrel{\text { p.a.e. }}{\rightarrow}} f$ if and only if $\mu$ has property $(P S)$.

Proof. Necesssity. Let be $\left(A_{n}\right)_{n} \subset \mathcal{A}$ such that $\lim _{n \rightarrow \infty} h\left(\mu\left(A \backslash A_{n}\right)\right.$, $\mu(A))=0$ and let us define for every $n \in \mathbb{N}$,

$$
f_{n}(x)=\left\{\begin{array}{ll}
1, & \text { if } x \in A_{n} \\
0, & \text { if } x \in A \backslash A_{n} .
\end{array} .\right.
$$

We see that $\left\{f_{n}\right\} \subset \mathcal{M}$ and $f_{n} \underset{A}{\stackrel{p . \mu}{\rightarrow}} 0$. Then there exists a subsequence $\left\{f_{n_{k}}\right\}_{k}$ of $\left\{f_{n}\right\}$ such that $f_{n_{k}} \underset{A}{\stackrel{p . a . e .}{\rightarrow}} 0$, whence $\mu(A)=\mu\left(A \backslash \bigcap_{m=1}^{\infty} \bigcup_{k=m}^{\infty}\{x \in\right.$ $\left.A ;\left|f_{n_{k}}(x)-0\right| \geq \varepsilon\right\}$ ), for every $\varepsilon>0$.

Particularly, for $\varepsilon=\frac{1}{2}$, we obtain $\mu(A)=\mu\left(A \backslash \bigcap_{m=1}^{\infty} \bigcup_{k=m}^{\infty} A_{n_{k}}\right)$, or, equivalently, $\mu(A)=\mu\left(\bigcup_{m=1}^{\infty} \bigcap_{k=m}^{\infty}\left(A \backslash A_{n_{k}}\right)\right)$, which says that $\mu$ has property (PS).

Sufficiency. Suppose that $\mu$ has property (PS) and $f_{n} \underset{A}{\stackrel{p \cdot \mu}{\rightarrow}} f$. Then we can find $\left\{n_{k}\right\}_{k}$ such that $\lim _{n \rightarrow \infty} h\left(\mu(A), \mu\left(A \backslash B_{k}\right)\right)=0$, where $B_{k}=\{x \in$ $\left.A ;\left|f_{n_{k}}(x)-f(x)\right| \geq \frac{1}{k}\right\}$, for $k \in \mathbb{N}^{*}$.

Since $\mu$ has property (PS), there exists a subsequence $\left(B_{k_{l}}\right)_{k}$ of $\left(B_{k}\right)_{k}$ such that $\mu(A)=\mu\left(A \backslash \bigcap_{m=1}^{\infty} \bigcup_{l=m}^{\infty} B_{k_{l}}\right)$. It is easy to see that $\left\{f_{n_{k_{m}}}\right\}_{m}$ converges to $f$ on the set $A \backslash \bigcap_{m=1}^{\infty} \bigcup_{l=m}^{\infty} B_{k_{l}}$, and, consequently, $f_{n_{k_{m}}} \underset{A}{\stackrel{\text { p.a.e. }}{\text {. }}}$ $f$.

From Theorem 4.5 and by Theorem 4.3 from [22], we obtain:

Corollary 4.6. If $\mu$ is a fuzzy multimeasure which satisfies properties $(P S)$ and $(P E), A \in \mathcal{A}, f \in \mathcal{M}$ and $\left\{f_{n}\right\} \subset \mathcal{M}$ are so that $f_{n} \underset{A}{\vec{p}} f$, then there exists a subsequence $\left\{f_{n_{k}}\right\}_{k}$ of $\left\{f_{n}\right\}$ such that $f_{n_{k}}^{\stackrel{p . a . u .}{\longrightarrow}} f$.

\section{Concluding remarks}

In this paper, we investigated for set-valued non-additive monotonic set functions, some relationships among the main types of convergences of sequences of measurable functions. 
In this way, we insisted on different types of pseudo-convergences of sequences of measurable functions, such as, pseudo-almost everywhere (p.a.e.), pseudo-almost uniform (p.a.u) convergences and pseudo-convergence in measure (p. $\mu$ ) and on the relationships among them, or with almost everywhere, almost uniform convergences and convergence in measure.

Thus, we obtained several set-valued versions of Lebesgue theorem and a pseudo-version of Riesz's theorem.

\section{REFERENCES}

1. Denneberg, D. - Non-Additive Measure and Integral, Theory and Decision Library, Series B: Mathematical and Statistical Methods, 27, Kluwer Academic Publishers Group, Dordrecht, 1994.

2. Gavriluţ, A. - Non-atomicity and the Darboux property for fuzzy and non-fuzzy Borel/Baire multivalued set functions, Fuzzy Sets and Systems, 160 (2009), 13081317.

3. HA, M.; WANG, X.; Wu, C. - Fundamental convergence of sequences of measurable functions on fuzzy measure space, Fuzzy Sets and Systems, 95 (1998), 77-81.

4. Hu, S.; PApageorgiou, N.S. - Handbook of Multivalued Analysis, Vol. I. Theory. Mathematics and its Applications, 419, Kluwer Academic Publishers, Dordrecht, 1997.

5. Jiang, Q.S.; Suzuki, H.; Wang, Z.Y.; KliR, G.J.; Li, J.; Yasuda, M. - Property (p.g.p.) of fuzzy measures and convergence in measure, J. Fuzzy Math., 3 (1995), 699-710.

6. Kawabe, J. - The Egoroff theorem for non-additive measure in Riesz spaces, Fuzzy Sets and Systems, 157 (2006), 2762-2770.

7. KawABE, J. - The Egoroff property and the Egoroff theorem in Riesz space-valued non-additive measure theory, Fuzzy Sets and Systems, 158 (2007), 50-57.

8. LI, G.; LI, J.; YASUdA, M. - Almost everywhere convergence of random set sequence of non-additive measure spaces, International Fuzzy Systems Association, Beijing, 2005.

9. LI, J. - Order continuous of monotone set function and convergence of measurable functions sequence, Appl. Math. Comput., 135 (2003), 211-218.

10. LI, J. - Egoroff's theorem on fuzzy measure spaces, Journal of Lanzhou University, 32 (1996), 19-22.

11. LI, J.; LI, J.Z. - A pseudo-version of Egoroff's theorem in non-additive measure theory, Mohu Xitong yu Shuxue, 21 (2007), 101-106. 
12. Li, J.; OUYAng, Y.; YASUdA, M. - Pseudo-convergence of measurable functions on Sugeno fuzzy measure space, Proc. of $7^{\text {th }}$ Conference on Information Science, North Carolina, USA, Sept. 26-30, 56-59.

13. Li, J.; YASUdA, M. - On Egoroff's theorem on finite monotone non-additive measure space, Fuzzy Sets and Systems, 153 (2005), 71-78.

14. Li, J.; Yasuda, M.; Jiang, Q.; Suzuki, H.; Wang, Z.; Klir, G.J. - Convergence of sequence of measurable functions on fuzzy measure spaces, Fuzzy Sets and Systems, 87 (1997), 317-323.

15. LIU, Y.-K. - On the convergence of measurable set-valued function sequence on fuzzy measure space, Fuzzy Sets and Systems, 112 (2000), 241-249.

16. Murofushi, T. - Duality and ordinality in fuzzy measure theory, Fuzzy Sets and Systems, 138 (2003), 523-535.

17. Murofushi, T.; UChino, K.; Asahina, S. - Conditions for Egoroff's theorem in non-additive measure theory, Fuzzy Sets and Systems, 146 (2004), 135-146.

18. PAP, E. - Null-Additive set Functions, Mathematics and its Applications, 337, Kluwer Academic Publishers Group, Dordrecht, Ister Science, Bratislava, 1995.

19. Precupanu, A. - Mathematical Analysis - Measure and Integration, I (in Romanian), Ed. Univ. "Al.I. Cuza", Iaşi, 2006.

20. Precupanu, A.; Gavriluț, A. - A set-valued Egoroff type theorem, Fuzzy Sets and Systems, 175 (2011), 87-95.

21. Precupanu, A.; Gavriluţ, A. - Set-valued Lusin type theorem for null-null-additive set multifunctions, Fuzzy Sets and Systems, 204 (2012), 106-116.

22. Precupanu, A.; Gavriluţ, A. - Pseudo-convergences of sequences of measurable functions on monotone multimeasure spaces, An. Ştiinţ. Univ. "Al.I. Cuza" Iaşi. Mat. (N.S.), 58 (2012), 67-84.

23. Ren, X.; Wu, C.; Wu, C. - Some remarks on the double asymptotic null-additivity of monotonic measures and related applications, Fuzzy Sets and Systems, 161 (2010), 651-660.

24. SonG, J.; LI, J. - Lebesgue theorems in non-additive measure theory, Fuzzy Sets and Systems, 149 (2005), 543-548.

25. Sun, Q.H. - Property $(S)$ of fuzzy measure and Riesz's theorem, Fuzzy Sets and Systems, 62 (1994), 117-119.

26. Sun, Q. - On the pseudo-autocontinuity of fuzzy measures, Fuzzy Sets and Systems, 45 (1992), 59-68.

27. Takahashi, M.; Asahina, S.; Murofushi, T. - Conditions for convergence theorems in non-additive measure theory, Faji Shisutemu Shinpoziumu Koen Ronbunshu, 21 (2005), 11-21.

28. Zhang, G. - Convergence of a fuzzy number, Fuzzy Sets and Systems, 57 (1993), $75-84$. 
29. WANG, Z.Y. - Asymptotic structural characteristics of fuzzy measures and their applications, Fuzzy Sets and Systems, 16 (1985), 277-290.

30. Wang, Z.Y.; KliR, G.J. - Fuzzy Measure Theory, Plenum Press, New York, 1992.

31. Wu, J.; LiU, H. - Autocontinuity of set-valued fuzzy measures and applications, Fuzzy Sets and Systems, 175 (2011), 57-64.

Received: 6.I.2011

Faculty of Mathematics, "Al.I. Cuza" University, Bd. Carol I, no 11, Iaşi, 700506, ROMANIA aprecup@uaic.ro

Faculty of Mathematics,

"Al.I. Cuza" University, Bd. Carol I, no 11, Iaşi, 700506,

ROMANIA

gavrilut@uaic.ro 